\title{
Is there implicit memory without attention? A reexamination of task demands in Eich's (1984) procedure
}

\author{
NOELLE L. WOOD, MICHAEL A. STADLER, and NELSON COWAN \\ University of Missouri, Columbia, Missouri
}

\begin{abstract}
The relation between memory and attention has been of long-standing interest. Eich (1984) made an important discovery of implicit but not explicit memory for contextually determined homophones (e.g., taxi-FARE) presented in a channel to be ignored within a selective listening procedure. However, his slow rate of presentation of shadowing task materials may have allowed frequent attention shifts to the allegedly ignored channel. With a direct replication of Eich's timing parameters, we reproduced his results, but when the attended channel was presented twice as fast as Eich's, implicit memory for the to-be-ignored words vanished. Our results contradict claims of extensive semantic processing of unattended auditory information in this task.
\end{abstract}

An important issue in the investigation of the limits of human cognition is the degree to which people process and remember events without paying attention to them. Considerable evidence exists that unattended information is not processed to such an extent that it subsequently will be available for tests of explicit memory such as free recall or recognition (Kidd \& Greenwald, 1988; Norman, 1969; Treisman, 1964a, 1964b). Explicit (or direct) memory tests may be contrasted with implicit (or indirect) memory tests, which are designed so that prior exposure to the tested information may influence subjects' performance on the test, even though no instructions are given asking subjects to recall that prior exposure explicitly. Implicit tests have often revealed memory in cases where there was thought to be none, as in the amnesic syndrome (see, e.g., Graf \& Schacter, 1985). Do implicit tests, in contrast with explicit ones, reveal evidence of memory for unattended information? Investigations of the effect of manipulating attention on implicit tests in which subjects study to-be-tested items while simultaneously performing a secondary, distracting task have provided positive results (see, e.g., Jacoby, Toth, \& Yonelinas, 1993; Parkin \& Russo, 1990). However, researchers have rarely asked subjects fully to ignore items that are to be tested, and then examined implicit memory for the ignored information.

An important, but isolated, exception is a report of implicit memory for supposedly unattended information by Eich (1984). He had subjects shadow, or repeat word for

This work was supported by NICHD Grant HD-21338 to N.C. We thank Eric Eich, Anthony Greenwald, John Kihlstrom, and Phil Merikle for helpful comments, and Dion Rigdon for assistance in data collection. Address correspondence to M. A. Stadler or N. Cowan, Department of Psychology, 210 McAlester Hall. University of Missouri, Columbia, MO 65211 (e-mail: psymike@showme.missouri. edu, psycowan@) showme.missouri.edu). word, a prose passage presented in one ear, while a list of critical word pairs was repeatedly presented in the other ear. Each of the word pairs consisted of a homophone preceded by a descriptive word that specified the less common of two possible meanings (e.g. taxi-FARE). Following the shadowing task, subjects displayed no explicit memory for the homophones alone (without the descriptor words) in a surprise recognition test. However, when asked to spell the same homophones in a test of implicit memory, subjects were more likely to give the less common spelling for the old homophones than they were for homophones that had not been presented during shadowing. This less common spelling corresponded to the context that had appeared on the to-be-ignored auditory channel (e.g., FARE instead of FAIR). These results were taken as evidence of implicit memory for the unattended homophones. This study has been cited more than 100 times. It may also be the only study that appears to meet what Greenwald (1992, p. 775) has called "the two-word challenge," described as "the task of demonstrating that attentionless unconscious cognition can extract the meaning of a two-word sequence."

We were concerned, however, that the rate of presentation of the materials in Eich's (1984) shadowing task was such that considerable attention to the to-be-tested homophones might have remained. This caused us (and Cowan, 1995) to question Eich's conclusion that he found implicit memory without attention. One problem is that the attended prose passage was presented at a very slow rate of 85 words per minute (wpm). In selective listening research, the attended passage is typically presented at 120-150 wpm (e.g., Moray, 1959; Treisman, 1964a, 1964c). The rate has been increased to $175 \mathrm{wpm}$ without substantially impairing shadowing efficiency (Wood \& Cowan, 1995). The slow rate of Eich's attended passage may have allowed frequent attention shifting to the to-beignored channel, without noticeable disruptions in subjects' shadowing performance. 
Table 1

Descriptor-Homophone Word Pairs

\begin{tabular}{|c|c|c|c|}
\hline \multicolumn{2}{|l|}{ Set IA } & \multicolumn{2}{|l|}{ Set IIA } \\
\hline taxi-FARE & $(.13)$ & pickled-BEET & $(.13)$ \\
\hline movie-REEL & $(.18)$ & opinion-POLL & $(.18)$ \\
\hline Bartlett-PEAR & $(.20)$ & ripped-SEAM & $(.18)$ \\
\hline deep-SEA & $(.20)$ & tall-TALE & $(.20)$ \\
\hline cotton-BALE & $(.28)$ & navy-PIER & $(.23)$ \\
\hline daily-MAIL & $(.30)$ & rubber-SOLE & $(.28)$ \\
\hline youngest-SON & $(.30)$ & savagely-MAUL & $(.32)$ \\
\hline last-WEEK & $(.33)$ & loud-GROAN & $(.40)$ \\
\hline \multicolumn{2}{|l|}{ Set IB } & \multicolumn{2}{|l|}{ Set IIB } \\
\hline $\operatorname{disc}-\mathrm{BRAKE}$ & $(.10)$ & thread-BARE & $(.13)$ \\
\hline easy-PREY & $(.13)$ & sprightly-GAIT & $(.13)$ \\
\hline window-PANE & $(.15)$ & right-HERE & $(.23)$ \\
\hline Harper's-FERRY & $(.20)$ & elastic-WAIST & $(.23)$ \\
\hline sneak-PEEK & $(30)$ & dramatic-ROLE & $(.25)$ \\
\hline prison-CELL & $(.32)$ & handily-wON & $(.25)$ \\
\hline garage-SALE & $(.35)$ & achilles'-HEEL & $(.33)$ \\
\hline strong-STEEL & $(.37)$ & sand-PAIL & $(.37)$ \\
\hline
\end{tabular}

Note-During the shadowing task, word pairs (as shown) were presented in the to-be-ignored channel. During the memory tests, only the homophones, shown here in capital letters, were presented. In Eich's (1984) to-be-ignored channel, the word Catalina was used instead of Harper's as shown here. This change was made because Catalina is longer and less uniformly familiar to subjects than the other descriptor words. Normative probabilities of spelling each homophone in the less common manner, based on Galbraith and Taschman's (1969) norms, are in parentheses above.

Similarly, the slow rate of presentation of the words in the to-be-ignored channel may have been detrimental to the maintenance of a singular focus of attention on the shadowed materials, instead drawing attention to the tobe-ignored channel. The rate was 1 word per second (wps) within a pair, with $2 \mathrm{sec}$ of silence between word pairs. The pauses between word pairs may have prevented habituation to the to-be-ignored channel. (For a discussion of attentional habituation, see Cowan, 1988, 1995.) Such habituation is presumably needed to assist subjects in focusing their attention exclusively on the attended channel.

In order to investigate the relation between attention and memory while addressing the concerns above, we carried out a direct replication of Eich (1984) but also tested conditions more favorable to focusing attention solely on the to-be-attended channel. In three different modifications, the rate of presentation of items in the attended channel, in the to-be-ignored channel, or in both channels was changed to double what Eich used.

Though we attempted as close a replication of Eich's (1984) procedure as we thought advisable, a few methodological improvements should be noted. The first pertains to Eich's use of half of the homophones from the shadowing task in the subsequent recognition test phase. Whereas Eich always used the same half of the homophones in the recognition phase, we counterbalanced the homophones presented in the recognition test. Thus, half of our subjects were recognition tested on the same items as Eich's subjects were, and half were tested on the words that he did not use in that phase. Second, whereas Eich always presented certain homophones in the shadowing phase, to be used later as memory targets (old words), and omitted certain others from the shadowing phase, to be used later as foils (new words), we counterbalanced the assignment of homophones to the "old" and "new" categories. (Our counterbalancing scheme is described completely in Tables 1 and 2, taken together.) Third, whereas Eich read the recognition and spelling lists aloud to each subject, we digitized, recorded, and played the test lists using a computer and audiotapes. The test list items were thus made acoustically identical to items in the to-be-ignored channel in order to maximize the similarity between study and test.

Table 2

Stimuli and Conditions of Eich (1984) and the Present Study

\begin{tabular}{|c|c|c|c|c|}
\hline \multirow[b]{2}{*}{ Group(s) } & \multicolumn{2}{|c|}{ Stimulus Sets Used } & \multicolumn{2}{|c|}{ Channel Speed } \\
\hline & Shadowing Phase & Recognition Test & Attended & Unattended \\
\hline \multicolumn{5}{|c|}{ Eich (1984) } \\
\hline & IA, IB & IA (old), IIA (new) & slow & slow \\
\hline & & The Present Study & & \\
\hline 1 & IA, IB & [A (old), IIA (new) & slow & slow \\
\hline 2 & IA, IB & IB (old), IIB (new) & slow & slow \\
\hline 3 & IIA, IIB & IIA (old), IA (new) & slow & slow \\
\hline 4 & IIA, IIB & IIB (old), IB (new) & slow & slow \\
\hline $5-8$ & \multicolumn{2}{|c|}{$\begin{array}{l}\text { (Stimulus words as in Groups } 1-4 \text {, } \\
\text { respectively) }\end{array}$} & slow & double \\
\hline $9-12$ & \multicolumn{2}{|c|}{$\begin{array}{l}\text { (Stimulus words as in Groups 1-4, } \\
\text { respectively) }\end{array}$} & double & slow \\
\hline $13-16$ & \multicolumn{2}{|c|}{$\begin{array}{l}\text { (Stimulus words as in Groups } 1-4, \\
\text { respectively) }\end{array}$} & double & double \\
\hline
\end{tabular}

Note-Each counterbalancing group included 6 subjects, for a total of 24 subjects per attentional condition or 96 subjects in all. Stimulus Sets IA, IB. IIA, and IIB refer to word sets listed in Table 1, though only the word in each pair shown there in capital letters was used in the recognition test. For each subject. all 32 of the capitalized words in Table 1 were used subsequently in the spelling test. Nonhomophonic filler items were also included in the experiment, as indicated in the text. The order of words in each phase was random. 
Table 3

Mean Performance Levels in All Conditions of the Present Study

\begin{tabular}{|c|c|c|c|c|c|c|c|c|}
\hline \multirow{2}{*}{$\begin{array}{l}\text { Attended } \\
\text { Channel }\end{array}$} & \multirow{2}{*}{$\begin{array}{l}\text { To-Be-Ignored } \\
\text { Channel }\end{array}$} & \multirow[b]{2}{*}{$N$} & \multicolumn{2}{|c|}{ Old } & \multicolumn{2}{|c|}{ New } & \multirow{2}{*}{$\begin{array}{c}\text { Memory } \\
\text { (Old - New) }\end{array}$} & \multirow{2}{*}{$\begin{array}{c}\text { Sensitivity } \\
\left(d^{\prime}\right)\end{array}$} \\
\hline & & & $M$ & $S D$ & $M$ & $S D$ & & \\
\hline \multicolumn{9}{|c|}{ Eich (1984): Recognition } \\
\hline Slow & slow & 16 & .40 & .24 & .38 & .24 & .02 & 0.06 \\
\hline \multicolumn{9}{|c|}{ Present Study: Recognition } \\
\hline Slow & slow & 24 & .48 & .27 & .50 & .28 & -.02 & -0.05 \\
\hline Slow & double & 24 & .54 & .25 & .44 & .23 & .10 & 0.25 \\
\hline Double & slow & 24 & .52 & .22 & .42 & .15 & .10 & 0.25 \\
\hline Double & double & 24 & .45 & .29 & .39 & .29 & .06 & 0.41 \\
\hline \multicolumn{9}{|c|}{ Eich (1984): Spelling } \\
\hline Slow & slow & 16 & .35 & $.17^{*}$ & .23 & $.16^{*}$ & .12 & 0.32 \\
\hline \multicolumn{9}{|c|}{ Present Study: Spelling } \\
\hline Slow & slow & 24 & .28 & .02 & .20 & .09 & .08 & 0.26 \\
\hline Slow & double & 24 & .31 & .07 & .25 & .10 & .06 & 0.18 \\
\hline Double & slow & 24 & .25 & .11 & .28 & .08 & -.03 & -0.09 \\
\hline Double & double & 24 & .23 & .09 & .25 & .12 & -.02 & -0.03 \\
\hline
\end{tabular}

Note-Means refer to proportion judged "old" (recognition) or spelled in accord with the context presented during shadowing (spelling). Each mean for the present study is collapsed across the four stimulus counterbalancing groups depicted in Table 2. Sensitivity $d^{\prime}$ scores are based on group means. "Eich (1984) reported separate SDs in the spelling test for tested and untested words. The estimates shown above are averages across both of these (old, .19 and .15; new, .17 and .14).

\section{METHOD}

\section{Subjects}

Ninety-six students (42 male, 54 female) were recruited from introductory psychology courses at the University of Missouri, Columbia. The subjects were native English speakers with no known hearing or speech impairments.

\begin{abstract}
Apparatus
All of the stimuli were initially recorded on an audiocassette deck by one female speaker, who read from a computer monitor that displayed the stimuli at the desired rates. These initial recordings were then transferred to a computer at a sampling rate of $22.05 \mathrm{kHz}$ with a dynamic resolution of 16 bits. The digitized recordings were used to construct sequences for the attended and to-be-ignored channels, and for the memory tests. The constructed channels were then synchronized and output to separate tracks of an audiocassette tape for selective listening, and to both tracks at once for the memory test lists.

The subjects were tested individually in a sound-attenuated chamber. Stimulus tapes were presented over audiological headphones. As in Eich's (1984) procedure, the attended channel was always presented to the right ear, at an intensity of approximately $72 \mathrm{~dB}$ (A) measured with a sound level meter and earphone coupler; the to-be-ignored channel was presented to the left ear, at approximately $64 \mathrm{~dB}(\mathrm{~A})$. The subjects shadowed into a microphone and were recorded on one track of a second tape deck, while the attended channel was transferred to the other track of that response tape deck. The memory test tapes each were presented binaurally at approximately $68 \mathrm{~dB}(\mathrm{~A})$.
\end{abstract}

\section{Stimuli and Procedure}

We used Eich's (1984) list of 32 descriptor-homophone word pairs, shown in Table 1. The normative probability of spelling a homophone in its less common way (e.g., FARE rather than FAIR) ranged from .10 to .40 as derived from Galbraith and Taschman's (1969) norms and averaged 240 for each of the two homophone lists. The number of letters for the two possible spellings of each homophone was constant. In the shadowing task, each presented homophone was paired with a descriptive word suggesting its less common spelling (e.g., taxi-FARE), as shown in the table. In the recognition test, only the words shown in capital letters were presented. Moreover, in keeping with Eich's design, not every item was used in every experimental phase for every subject (though they were all used for every subject in the last phase, the spelling test). Table 2 depicts the words used in every phase of the experiment conducted by Eich (1984) and in our more completely counterbalanced and extensive experiment.

Shadowing task. The subjects were informed that the primary purpose of the experiment was to determine how well people are able to comprehend a story while being distracted by other sounds. The subjects were asked to shadow the story presented to their right ear and ignore the distracting sounds presented to their left ear. The attended channel consisted of a $10-$ min passage from the humorous essay (Trillin, 1982) used by Eich (1984), recorded at one of two rates: Eich's rate of $85 \mathrm{wpm}$, or $170 \mathrm{wpm}$.

There were two speeds of presentation of the to-be-ignored channel: the rate used by Eich (1984), and twice that rate. Regardless of rate, the to-be-ignored channel materials were presented in the same overall order as were Eich's. Specifically, the presentation comprised the following, in this sequential order: (1) the alphabet from A to Z, once; (2) 4 noncritical buffer pairs consisting of a modifier and a nonhomophone (e.g., hilton-HOTEL); (3) 16 critical descriptor-homophone pairs (e.g., taxi-FARE) repeated 8 times, in a new random order each time; (4) 4 other noncritical buffer pairs; and (5) the alphabet once more.

Repetitions of the descriptor-homophone pairs were all exact duplicates of the originally digitized pairs. In Eich's (1984) condition, the alphabet was presented at 1 letter per second, and both the noncritical buffer pairs and the descriptor-homophone pairs were presented at 1 pair per $4 \mathrm{sec}$ (specifically, $1 \mathrm{sec}$ per word in a pair, followed by 2 sec of silence between pairs).

In the double-speed condition, the timing of events in the to-beignored channel was altered to increase their uniformity and therefore minimize the likelihood of attention shifts to this channel when the critical word pairs began. The alphabet still was presented at an average rate of 1 letter per second, but letters were grouped into pairs taking up $1 \mathrm{sec}$, with $1 \mathrm{sec}$ of silence between pairs. The word pairs, like the letters, were presented at the overall rate of 1 pair per $2 \mathrm{sec}$, twice Eich` (1984) rate. Each word pair 


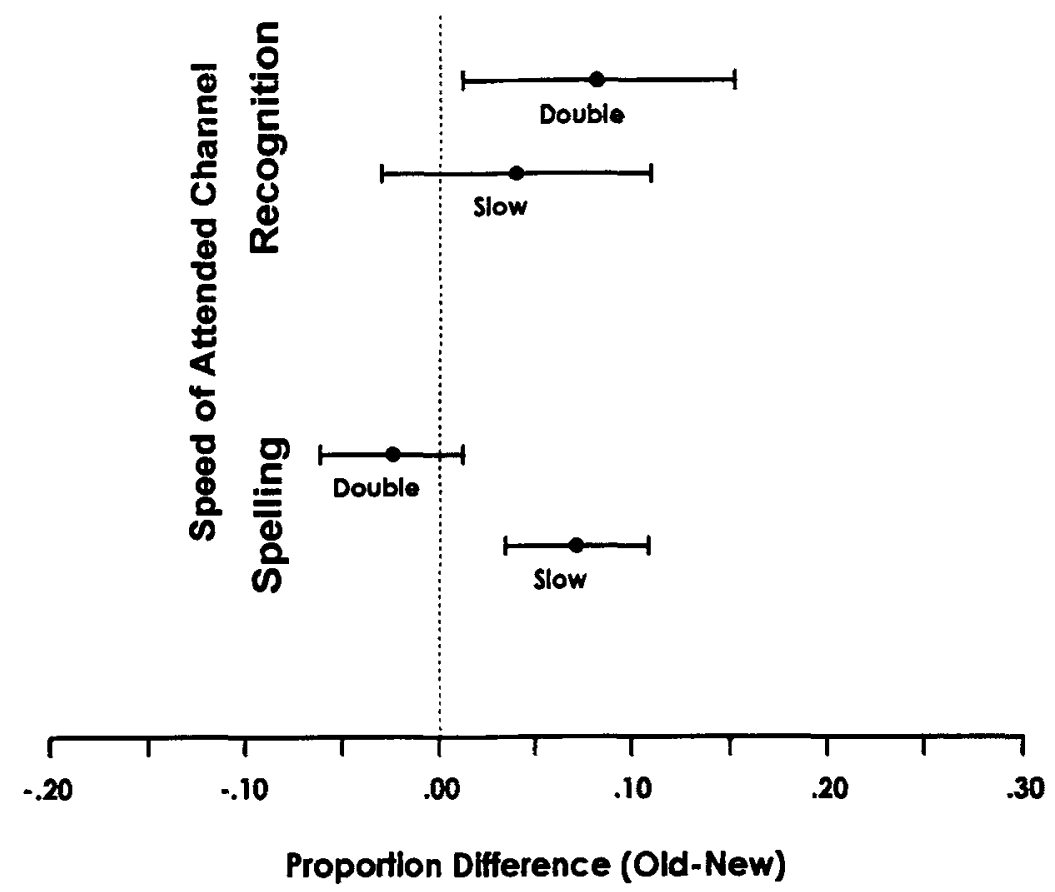

Figure 1. Recognition and spelling test scores expressed as the proportion difference between words that were old (presented during the shadowing task) versus new (not presented), as a function of the rate of presentation of materials in the attended channel in the shadowing task. Each data point is based on $\mathbf{4 8}$ subjects. Error bars indicate $95 \%$ confidence intervals.

took up $1.2-1.3 \mathrm{sec}$, complemented by $700-800 \mathrm{msec}$ of silence between pairs. To fill the channel, the new rate required that all 8 (rather than 4) noncritical buffer pairs be repeated both before and after the critical pairs, and that the critical-pair list be repeated 16 times (rather than 8 ).

The channels that were to be attended and ignored were presented concurrently. The attended channel always began $2,650 \mathrm{msec}$ before and ended $2,650 \mathrm{msec}$ after the to-be-ignored channel. Each subject received one of four combinations of attended and to-be-ignored channel speed conditions: both channels as slow as Eich's (1984), the attended channel doubled in speed, the to-beignored channel doubled in speed, or both channels doubled (see Table 2)

Memory tests. Following the shadowing task, all subjects gave a $1-$ min spoken synopsis of the attended story. The subjects were then informed that a secondary purpose of the research was to determine whether they had any memory for the content of the tobe-ignored channel.

Explicit (recognition) and implicit (spelling) memory test words were presented at a rate of 1 wps with $4 \mathrm{sec}$ of silence between words, during which time the subjects responded. The subjects' verbal responses were recorded by the experimenter. For the test of explicit memory, the subjects were to listen carefully to each word in the list, decide whether that word was "old" (previously presented in the to-be-ignored channel during shadowing) or "new," and rate their confidence in their decision on a scale of 1-6 $(1=$ definitely old, $2=$ probably old, $3=$ guess old, $4=$ guess new, $5=$ probably new, $6=$ definitely new). This recognition test list included 8 old and 8 new homophones (as well as 16 filler items). selected in the same manner as were Eich's (1984).

For the test of implicit memory, the subjects were asked to spell each word that they heard with the first spelling that came to mind. As in Eich's (1984) procedure, only one randomly ordered list of words was constructed for the spelling test. The list contained 16 old homophones and 16 new homophones (i.e., all 32 items shown in capital letters in Table 1), as well as 32 nonhomophone filler items. Half of the old and half of the new homophones in the spelling test previously had been presented in the recognition test. Although all subjects received the same spelling test list, the status of each homophone (previously tested vs. untested in the recognition test) varied across subjects according to the counterbalancing scheme shown in Table 2 .

\section{RESULTS}

We expected that the subjects might show implicit memory (but not explicit memory) for meaningful information presented under conditions of distraction, but not when attention was more strongly focused away from that information. Specifically, we anticipated implicit memory for homophones biased by descriptor words presented in the to-be-ignored channel of a selective listening task in the condition in which the rates of presentation of the shadowed and to-be-ignored materials were relatively slow, as in Eich's (1984) procedure. Such rates presumably would allow subjects' attention to shift rapidly between the shadowed and to-be-ignored materials without interrupting shadowing performance, allowing some attention to be paid to the subsequently tested homophones. In contrast, we anticipated that there might be no implicit or explicit memory for those same homophones under conditions in which attention was more fully directed away from them through the use of stimulus 


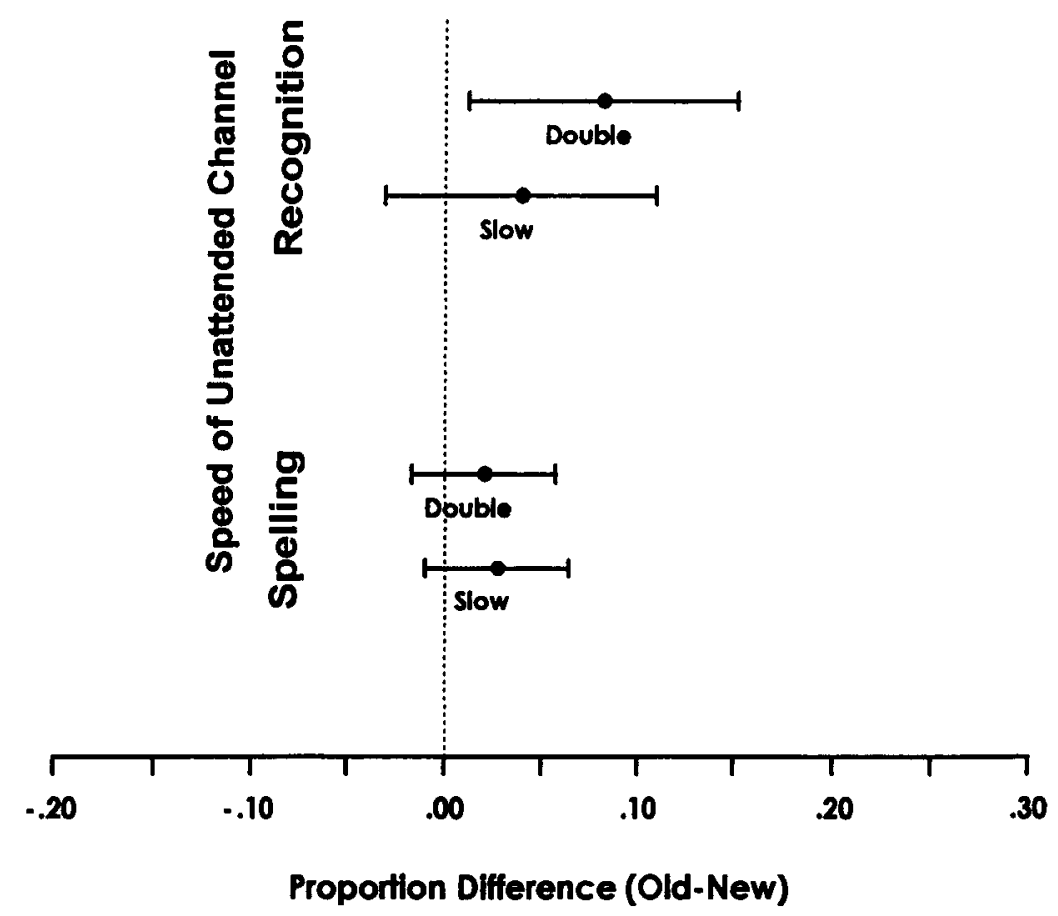

Figure 2. Recognition and spelling test scores expressed as the proportion difference between words that were old (presented during the shadowing task) versus new (not presented), as a function of the rate of presentation of materials in the channel to be ignored in the shadowing task. Each data point is based on 48 subjects. Error bars indicate $95 \%$ confidence intervals.

materials in which the shadowed channel, the to-beignored channel, or both channels were presented at twice the speed.

The mean scores in all conditions of this experiment, as well as those of Eich (1984) for the sake of comparison, are reported in Table 3 . The figures, however, depict difference scores obtained for homophone items by subtracting a proportion obtained for new items (those not presented in the unattended channel of the shadowing task) from a proportion obtained for old items (those presented in shadowing). For the recognition test, the scores that were involved were the proportions of items judged to have been presented in the shadowing task (confidence ratings of 3 or less); whereas for the spelling test, they were the proportions of items spelled according to the less common of two lexical interpretations (which, for the old items, matched the context that was presented in the shadowing task). For either type of test, a difference score reliably greater than 0 indicates memory of the unattended materials. The figures also show a $95 \%$ confidence interval for each data point.

Figure 1 shows the recognition and spelling test scores as a function of the rate of presentation of materials in the attended channel in the prior shadowing task, collapsed across other factors. With a slow presentation rate, we replicate the result obtained by Eich (1984). Specifically, the recognition test difference score does not reliably differ from 0 , whereas the spelling test score clearly is greater than 0 . With a faster presentation of the attended channel's materials, the results are quite different. The recognition test score may be slightly greater than 0 (though that is not certain), but the spelling test score no longer can be shown to differ from 0 . The most important finding here is that doubling the speed of the attended channel eliminates the tendency to spell words according to the context that had been presented in the unattended channel in the shadowing task, as we anticipated.

This demonstrated limitation in the generality of Eich's (1984) finding cannot be attributed to a difference in the statistical method of assessment. For example, analyses of variance of the spelling test scores revealed a significant difference between old and new items for the subjects who received a slow attended channel $[F(1,92)=$ $\left.17.48, M S_{\mathrm{e}}=.007, p<.001\right]$, but not for the subjects who received a fast attended channel $\left[F(1,92)=2.03, M S_{\mathrm{e}}=\right.$ $.007, p>.1]$.

Figure 2 is similar to Figure 1, except that the rates of presentation of the unattended (rather than the attended) materials in the shadowing task are examined. Once more, there is a hint that a fast presentation may allow some memory in the recognition task. However, overall there is no evidence of memory in the spelling task, with either presentation rate of unattended materials.

Each of the data points in Figures 1 and 2 was based on means across 48 subjects receiving a particular speed of presentation of materials in the channel examined. AIthough this method of data presentation maximizes the reliability of the result, it does not allow for possible in- 


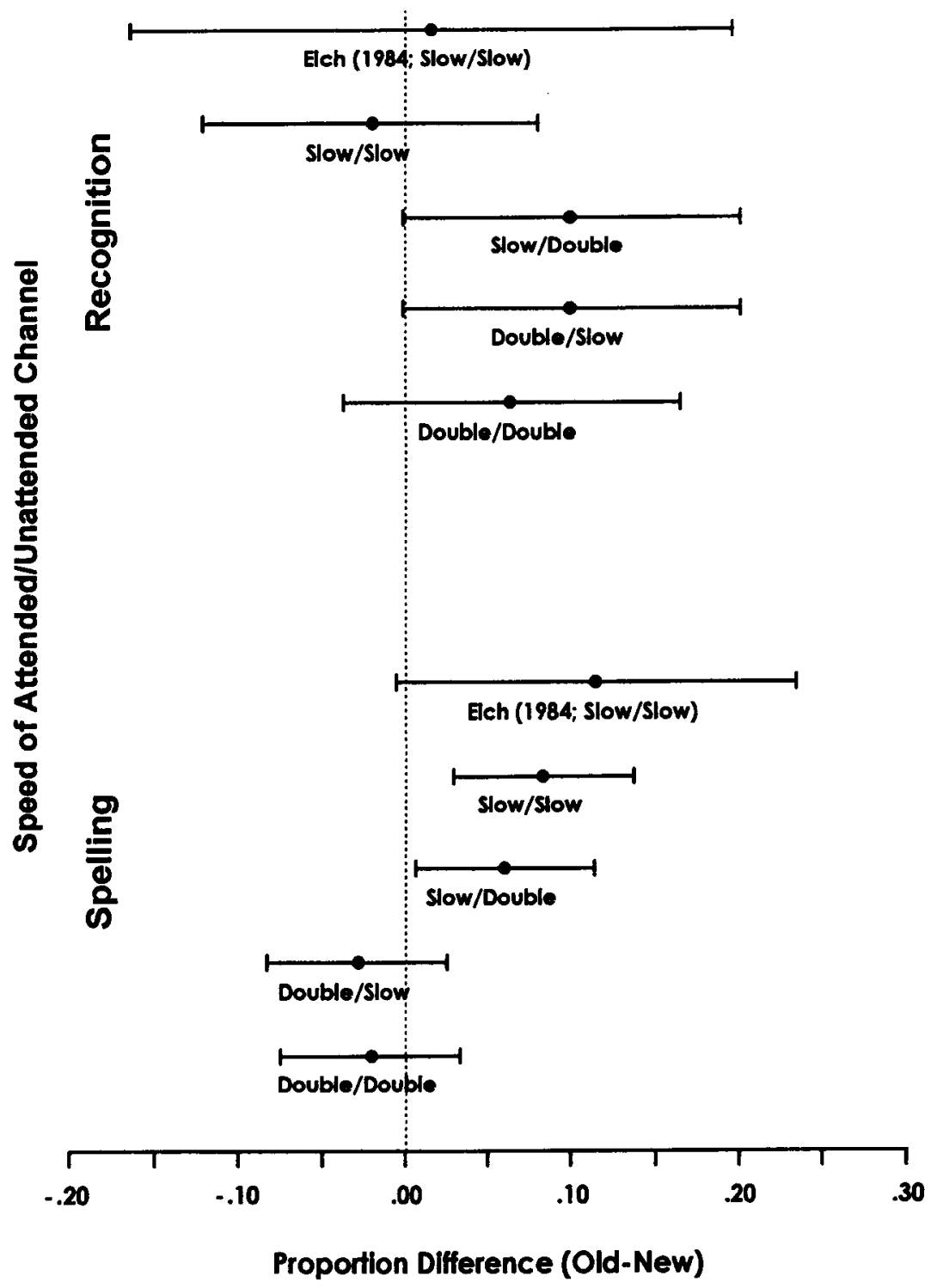

Figure 3. Recognition and spelling test scores expressed as the proportion difference between words that were old (presented during shadowing) versus new (not presented), as a function of the rate of presentation of materials in both channels of the shadowing task. Data from Eich (1984) are also presented for the sake of comparison. Each data point from Eich is based on 16 subjects, and each data point from the present experiment is based on 24 subjects. Error bars indicate $95 \%$ confidence intervals.

teractions between the speeds of materials in the attended and unattended channels. To gain a more detailed view, Figure 3 shows the data for each combination of attended and unattended channel speeds, both for recognition (top half of figure) and for spelling (bottom half). The means from Eich (1984) also are included. The top half of this figure shows that there may well have been recognition of the unattended materials when the rate of either channel was doubled. The bottom half of the figure clearly shows implicit memory of the unattended materials as measured in the spelling test, but only when the unattended channel materials were presented slowly in the shadowing task

For the recognition test, similar results were obtained in a more detailed examination in which we recalculated the (old - new) difference scores using mean confidence ratings for each condition. With that scoring method, negative numbers imply memory for old items. The means and $95 \%$ confidence intervals were, for attended and unattended presentation rates both slow, $0.06 \pm 0.34$; for attended slow and unattended fast, $-0.23 \pm 0.27$; for attended fast and unattended slow, $-0.30 \pm 0.28$; and for 
both fast, $-0.19 \pm 0.25$. Across all 96 subjects, the result was $-0.17 \pm .14$, suggesting that there was in fact some recognition of to-be-ignored items.

\section{DISCUSSION}

The central question that we asked was whether there is implicit memory for truly unattended information, and our findings, contrary to the conclusion of Eich (1984), suggest that the answer is "no." We did find implicit memory for homophones presented in a to-be-ignored channel under the slow presentation rate conditions that Eich used, but we suggest that this occurred because subjects under such conditions were able to pay at least partial attention to the to-be-ignored channel. With a faster rate of presentation in the attended channel, which controls attention much better, this finding was eliminated. Our results indicate the possibility of implicit memory for information presented under conditions of partial attention, but discredit the notion that implicit memory is revealed under conditions in which little or no attention is paid to the to-be-tested materials at the time of encoding.

We are not sure why a hint of memory emerged in the recognition task when either channel speed was doubled. However, the finding may be related to the fact that the recognition test required only that the acoustic form of old words seem more familiar than new words, rather than requiring identification of which homophonic variation was presented (as in the spelling test).

The homophonic variation can be identified only if the homophone and its context have been processed together, constraining the identification lexically or semantically, during shadowing. In terms of concepts commonly used in the literature, the recognition task may have required more data-driven processing and less conceptually driven processing than did the spelling task (see Jacoby, 1983; Roediger, Weldon, \& Challis, 1989). Speeding up the unattended channel could have assisted data-driven processing because each homophone was presented twice as many times as in the slow condition, and speeding the attended channel could have caused a greater reliance on data-driven processing because the better control of attention prevented conceptually driven processing of the unattended channel materials. It is worth noting, in any case, that Eich's 95\% confidence interval for recognition was wide enough to encompass all of our recognition means.

Although Eich's (1984) conclusion of implicit memory without attention may be questioned, his experiment, as well as our direct replication of that experiment, did reveal an interesting dissociation between implicit and explicit memory. Eich's experiment is important, in that it was one of the first to report that disrupting attention during encoding may result in memory on a potentially more sensitive test of implicit memory, but not on a potentially less sensitive test of explicit memory. Similar dissociations have been found by decreasing the attentional allocation during study (Jacoby et al., 1993;
Merikle \& Reingold, 1991; Parkin \& Russo, 1990), or by decreasing the duration of initial item exposure (KunstWilson \& Zajonc, 1980).

Provided that the explicit and implicit tests involve identical stimuli and differ only in instructions, a finding of better performance on the implicit memory test has been taken to indicate the operation of unconscious memory processes (Merikle \& Reingold, 1991; Reingold \& Merikle, 1988). However, this refers to memory in the absence of awareness of the prior stimulus presentation at the time of test. A stimulus could be encoded with some attention, but not enough to allow awareness of it at the time of test. Eich (1984) assumed that in his selective listening procedure, the to-be-tested homophones (1) were initially perceived without attention, or unconsciously, and (2) subsequently were remembered unconsciously in the implicit memory tests. It is only with the former assumption (1) that we take issue. Subjects may well devote some attention to the to-be-ignored stimuli in Eich's condition, yet still not process these stimuli fully enough to permit awareness of the prior presentation during the memory tests.

Holender (1986) cautioned that evidence of deep processing of to-be-ignored materials may be due to attention shifts to those materials that go undetected by the experimenter. In keeping with his suggestion, implicit memory may be evidenced in situations of distracted attention (see, e.g., Eich, 1984; Jacoby et al., 1993), but does not seem to occur when attention is strongly directed away from the materials to be tested (see also Wood \& Cowan, 1995). It appears that for the present situation involving the integration of a descriptor and a target homophone, one must have at least partially attended to the stimuli in order to have a meaningful memory representation of them. It remains to be seen whether there is a more rudimentary form of implicit memory for stimuli that are totally unattended at the time of their presentation, such as that indexed by simple semantic priming.

\section{REFERENCES}

Cowan, N. (1988). Evolving conceptions of memory storage, selective attention, and their mutual constraints within the human information processing system. Psychological Bulletin, 104, 163-191.

Cowan, N. (1995). Attention and memory: An integrated framework (Oxford Psychology Series, No. 26). New York: Oxford University Press.

EICH, E. (1984). Memory for unattended events: Remembering with and without awareness. Memory \& Cognition, 12, 105-111.

Galbraith, G. G., \& Taschman, C. S. (1969). Homophone units: A normative and methodological investigation of the strength of component elements. Journal of Verbal Learning \& Verbal Behavior, 8 , 737-744.

Graf, P., \& Schacter, D. L. (1985). Implicit and explicit memory for new associations in normal and amnesic subjects. Journal of Experimental Psychology: Learning, Memory \& Cognition, 11, 501-518.

Greenwald, A. G. (1992). New Look 3: Unconscious cognition reclaimed. American Psychologist, 47, 766-779.

Holender, D. (1986). Semantic activation without conscious identification in dichotic listening, parafoveal vision, and visual masking: A survey and appraisal. Behavioral \& Brain Sciences, 9, 1-66.

JACOBY, L. L. (1983). Remembering the data: Analyzing interactive 
processes in reading. Journal of Verbal Learning \& Verbal Behavior, 22, 485-508.

Jacoby, L. L., Toth, J. P., \& Yonelinas, A. P. (1993). Separating conscious and unconscious influences of memory: Measuring recollection. Journal of Experimental Psychology: General, 122, 139-154

KIDD, G. R., \& GREENWALD, A. G. (1988). Attention, rehearsal, and memory for serial order. American Journal of Psychology, 101, 259-279.

KUNST-WILSON, W. R., \& ZAJONC, R. B. (1980). Affective discrimination of stimuli that cannot be recognized. Science, 207, 557-558.

MerikLe, P. M., \& Reingold, E. M. (1991). Comparing direct (explicit) and indirect (implicit) measures to study unconscious memory. Journal of Experimental Psychology: Learning, Memory, \& Cognition, 17, 224-233.

MORAY, N. (1959). Attention in dichotic listening: Affective cues and the influence of instructions. Quarterly Journal of Experimental Psychology, 11, 56-60.

Norman, D. A. (1969). Memory while shadowing. Quarterly Journal of Experimental Psychology, 21, 85-93.

Parkin, A. J., \& Russo, R. (1990). Implicit and explicit memory and the automatic/effortful distinction. European Journal of Psychology, 2, 71-80.

REINGOLD, E. M., \& MERIKLE, P. M. (1988). Using direct and indirect measures to study perception without awareness. Perception \& Psychophysics, 44, 563-575.

Roediger, H. L., III, Weldon, M. S., \& Challis, B. H. (1989). Explaining dissociations between implicit and explicit measures of retention: A processing account. In H. L. Roediger III \& F. I. M. Craik (Eds.), Varieties of memory and consciousness: Essays in honour of Endel Tulving (pp. 3-41). Hillsdale, NJ: Erlbaum.

Treisman, A. M. (1964a). The effect of irrelevant material on the efficiency of selective listening. American Journal of Psychology, 77 533-546.

Treisman, A. M. (1964b). Selective attention in man. British Medical Bulletin, 20, 12-16.

Treisman, A. M. (1964c). Verbal cues, language, and meaning in selective attention. American Journal of Psychology, 77, 206-219.

TriLlin, C. (1982, April 12). U.S. journal: Pembroke Park, Florida. New Yorker, 58(8), 134, 136, 139-143.

WoOD, N. L., \& CowAN, N. (1995). The cocktail party phenomenon revisited: Attention and memory in the classic selective listening procedure of Cherry (1953). Journal of Experimental Psychology: General, 124, 243-262.

(Manuscript received June 11, 1996; revision accepted for publication November 19,1996 .) 